\title{
THE TRANSLATIONAL HULL OF A COMPLETELY 0-SIMPLE SEMIGROUP
}

\author{
by MARIO PETRICH
}

(Received 14 November, 1966)

Introduction. The translational hull $\Omega(S)$ of a semigroup $S$ plays an important role in the theory of ideal extensions of semigroups. In fact, every ideal extension of $S$ by a semigroup $T$ with zero can be constructed using a certain partial homomorphism of $T \backslash 0$ into $\Omega(S)$; a particular case of interest is when $S$ is weakly reductive (see $\$ 4.4$ of [3], [2], [7]). A theorem of Gluskin [6, 1.7.1] states that if $S$ is a weakly reductive semigroup and a densely embedded ideal of a semigroup $Q$, then $Q$ and $\Omega(S)$ are isomorphic. A number of papers of Soviet mathematicians deal with the abstract characteristic (abstract semigroup, satisfying certain conditions, isomorphic to the given semigroup) of various classes of (partial) transformation semigroups in terms of densely embedded ideals (see, e.g., [4]). In many of the cases studied, the densely embedded ideal in question is a completely 0-simple semigroup, so that Gluskin's theorem mentioned above applies. This enhances the importance of the translational hull of a weakly reductive, and in particular of a completely 0-simple semigroup. Gluskin [5] applied the theory of densely embedded ideals (which are completely 0 -simple semigroups) also to semigroups and rings of endomorphisms of a linear manifold and to certain classes of abstract rings.

It then seems evident that a great number of very different results can be brought under the common roof of the translational hull of a completely 0 -simple semigroup. This point of view promises a variety of new results not only in the theory of semigroups (e.g., construction of ideal extensions, representation of arbitrary or regular semigroups, embedding, the structure of various classes of binary relations on a set, etc.) but also in other branches of algebra (as pioneered by Gluskin [5] in the theory of semigroups and. rings of endomorphisms of a linear manifold). It is very likely that, with the further study of this subject, new applications in various fields will be found.

The purpose of this work is to construct a faithful representation of the semigroups of left and right translations of a completely 0-simple semigroup $S$, study its translational hull, and derive certain of its properties. These will be useful in the applications to the study of binary relations, semigroups of endomorphisms of a linear manifold, etc., which will be treated in subsequent communications.

Summary. In $\$ 1$ we construct a faithful representation of the semigroup of left [right] translations of a completely 0 -simple semigroup $S$ by means of pairs of functions; this is then used to characterize its translational hull $\Omega(S)$. In this characterization of $\Omega(S)$, we find necessary and sufficient conditions on an element in order that it shall correspond to an element of $\Omega(S)$ of the form $\left(\lambda_{a}, \rho_{a}\right)$. $\S 2$ is devoted to the comparison of the construction in $\S 1$ with the representation of left and right translations by means of matrices due to Clifford. $\$ 3$ deals with the conditions on $S$ that are necessary and sufficient in order that, e.g., to every left translation of $S$ there is at most one linked right translation of $S$. Finally, in $\S 3$ we discuss some properties of the concepts studied in $\$ 1$. 
Notation. Throughout the whole paper, $S$ denotes a completely 0 -simple semigroup with the Rees representation $S=\mathscr{M}^{0}(G ; I, M ; P) ; 1$ denotes the identity of the group $G$. We write left [right] translations as operators on the left [right]; $\Lambda(S)[\mathrm{P}(S)]$ denotes the semigroup of all left [right] translation of $S$ under multiplication: $\left(\lambda \lambda^{\prime}\right) x=\lambda\left(\lambda^{\prime} x\right)\left[x\left(\rho \rho^{\prime}\right)\right.$ $\left.=(x \rho) \rho^{\prime}\right]$ for all $x \in S$. The translations $\lambda \in \Lambda(S), \rho \in \mathrm{P}(S)$ are linked if $x(\lambda y)=(x \rho) y$ for all $x, y \in S ; \Omega(S)$ denotes the translational hull of $S$, that is, the subsemigroup of $\Lambda(S) \times \mathrm{P}(S)$ consisting of all pairs of linked translations. The subsemigroup of $\Omega(S)$ consisting of all pairs of the form $\left(\lambda_{a}, \rho_{a}\right)$ (recall, $\lambda_{a} x=a x, x \rho_{a}=x a$ for all $x \in S$ ) is denoted by $\Pi(S)$; the mapping $a \rightarrow\left(\lambda_{a}, \rho_{a}\right)$ is an isomorphism of $S$ onto $\Pi(S)$ since $S$ is weakly reductive; further, $\Pi(S)$ is a minimal ideal of $\Omega(S)$.

Let $K$ be a non-empty set. A function $\gamma$ of a subset of $K$ (called the domain of $\gamma$ and denoted by $\mathbf{d} \gamma$ ) onto a subset of $K$ (called the range of $\gamma$ and denoted by $r \gamma$ ) is called a partial transformation on $K$. The cardinality of $r \gamma$ is called the rank of $\gamma$ and denoted by rank $\gamma$. The set of all partial transformations on $K$ written as operators on the left under multiplication:

$$
\begin{gathered}
\mathrm{d}\left(\gamma \gamma^{\prime}\right)=\left\{x \in K \mid x \in \mathrm{d} \gamma^{\prime}, \gamma^{\prime} x \in \mathrm{d} \gamma\right\}, \\
\gamma \gamma^{\prime} x=\gamma\left(\gamma^{\prime} x\right) \text { if } \quad x \in \mathrm{d}\left(\gamma \gamma^{\prime}\right),
\end{gathered}
$$

is a semigroup denoted by $W(K)$. The set of all partial transformations on $K$ written as operators on the right under multiplication:

$$
\begin{aligned}
& \mathrm{d}\left(\gamma \gamma^{\prime}\right)=\left\{x \in K \mid x \in \mathrm{d} \gamma, x \gamma \in \mathrm{d} \gamma^{\prime}\right\}, \\
& x \gamma \gamma^{\prime}=(x \gamma) \gamma^{\prime} \text { if } \quad x \in \mathrm{d}\left(\gamma \gamma^{\prime}\right),
\end{aligned}
$$

is a semigroup denoted by $W^{\prime}(K)$. (Note that the identity mapping on the set $W(K)$ is an antiisomorphism of $W(K)$ onto $W^{\prime}(K)$.)

The zero in any semigroup is denoted by 0 ; e.g., the zero of $\Lambda(S)$ or $\mathrm{P}(S)$ is the transformation mapping $S$ onto its zero, the zero of $\Pi(S)$ and $\Omega(S)$ is the pair $(0,0)$, while the zero of $W(K)$ and $W^{\prime}(K)$ is the empty partial transformation (thus $\mathbf{d} 0=\mathbf{r} 0=\square$ ).

For all concepts and notation not defined in the paper, the reader is referred to [3]. We write functions as operators on either left or right as it is convenient in a particular case. Multiplication is always (except in the proof of Proposition 1) denoted by juxtaposition.

1. Basic results. Let $I$ be a non-empty set (elements of $I$ are denoted by $i, j, k, \ldots$ ) and let $G$ be a group. Set

$$
L(I, G)=\{(\alpha, \phi) \mid \alpha \in W(I), \alpha \neq 0, \phi: \mathrm{d} \alpha \rightarrow G\} \cup 0
$$

with multiplication

where

$$
\begin{aligned}
(\alpha, \phi)\left(\alpha^{\prime}, \phi^{\prime}\right) & \left.=\left(\alpha \alpha^{\prime}, \phi^{\prime \prime}\right) \text { if } \alpha \alpha^{\prime} \neq 0, \text { and } 0 \text { otherwise, }\right\} \\
(\alpha, \phi) 0 & =0(\alpha, \phi)=0,
\end{aligned}
$$

$$
\phi^{\prime \prime} i=\left(\phi \alpha^{\prime} i\right)\left(\phi^{\prime} i\right) \quad\left(i \in \mathrm{d}\left(\alpha \alpha^{\prime}\right)\right),
$$

(cf. [1], (4.4)). Observe that in $(\alpha, \phi)$ both $\alpha$ and $\phi$ are written as operators on the left. For every $\lambda \in \Lambda(S), \lambda \neq 0$, let 
where

$$
\mathbf{a} \lambda=(\alpha, \phi), \quad \mathbf{a} 0=0,
$$

$$
\lambda(1 ; i, \mu)=(\phi i ; \alpha i, \mu) \text { if } \lambda(1 ; i, \mu) \neq 0 .
$$

THEOREM 1. The function a is an isomorphism of $\Lambda(S)$ onto $L(I, G)$.

Proof. If $\lambda(1 ; i, \mu) \neq 0$, then $p_{\mu j} \neq 0$ for some $j \in I$ and

$$
\lambda(1 ; i, \mu)=\lambda\left[(1 ; i, \mu)\left(p_{\mu j}^{-1}, j, \mu\right)\right]=[\lambda(1 ; i, \mu)]\left(p_{\mu j}^{-1}, j, \mu\right) \neq 0 ;
$$

so $\lambda(1 ; i, \mu)=(c ; l, \mu)$ for some $c \in G, l \in I$ (that is, a left translation does not change $\mu$ ). If also $\lambda(1 ; i, v)=(d ; k, v)$, then $p_{\mu m} \neq 0$ for some $m \in I$ and

$$
\begin{aligned}
(c ; l, v) & =(c ; l, \mu)\left(p_{\mu m}^{-1} ; m, v\right)=[\lambda(1 ; i, \mu)]\left(p_{\mu m}^{-1} ; m, v\right) \\
& =\lambda\left[(1 ; i, \mu)\left(p_{\mu m}^{-1} ; m, v\right)\right]=\lambda(1 ; i, v)=(d ; k, v),
\end{aligned}
$$

whence $c=d, l=k$, that is to say, the functions $\alpha$ and $\phi$ defined by (5) do not depend on $\mu$ and are thus single-valued. Further, from (5) it follows that

$$
\mathrm{d} \alpha=\{i \in I \mid \lambda(1 ; i, \mu) \neq 0\},
$$

and it is easy to see that in fact $i \in \mathrm{d} \alpha$ if and only if $\lambda(a ; i, \mu) \neq 0$, where $a \in G$ and $\mu \in \mathrm{M}$ are arbitrary. It is now clear that $\alpha \in W(I)$ and $\phi: \mathrm{d} \alpha \rightarrow G$; thus a maps $\Lambda(S)$ into $L(I, G)$. $\alpha^{\prime} i \in \mathrm{d} \alpha$,

Let $\lambda, \lambda^{\prime} \in \Lambda(S)$ be different from zero and $\mathrm{a} \lambda=(\alpha, \phi), \mathrm{a} \lambda^{\prime}=\left(\alpha^{\prime}, \phi^{\prime}\right)$. Then, if $i \in \mathrm{d} \alpha^{\prime}$,

$$
\lambda\left[\lambda^{\prime}(1 ; i, \mu)\right]=\lambda\left(\phi^{\prime} i ; \alpha^{\prime} i, \mu\right)=\left(\left(\phi \alpha^{\prime} i\right)\left(\phi^{\prime} i\right) ; \alpha \alpha^{\prime} i, \mu\right)=\left(\phi^{\prime \prime} i ; \alpha \alpha^{\prime} i, \mu\right),
$$

where $\phi^{\prime \prime}$ is given by (3), while otherwise $\lambda\left[\lambda^{\prime}(1 ; i, \mu)\right]=0$. Consequently a $\left(\lambda \lambda^{\prime}\right)=\left(\alpha \alpha^{\prime}, \phi^{\prime \prime}\right)$ if $\alpha \alpha^{\prime} \neq 0$, and is 0 otherwise. Comparison of this with (2) proves that $(\mathbf{a} \lambda)\left(\mathbf{a} \lambda^{\prime}\right)=\mathbf{a}\left(\lambda \lambda^{\prime}\right)$ for non-zero $\lambda, \lambda^{\prime}$. Since the last equation is clearly valid when $\lambda=0$ or $\lambda^{\prime}=0$, we conclude that $\mathbf{a}$ is a homomorphism. It is easy to see that $\mathbf{a} \lambda=\mathbf{a} \lambda^{\prime}$ implies that $\lambda=\lambda^{\prime}$.

To show that a is onto, let $(\alpha, \phi) \in L(I, G)$. Define $\lambda: S \rightarrow S$ by

$$
\left.\begin{array}{l}
\lambda(a ; i, \mu)=((\phi i) a ; \alpha i, \mu) \text { if } i \in \mathrm{d} \alpha, \text { and } 0 \text { otherwise, } \\
\lambda 0=0 .
\end{array}\right\}
$$

Then, if $i \in \mathrm{d} \alpha, p_{\mu j} \neq 0$, we have

$$
\begin{aligned}
{[\lambda(a ; i, \mu)](b ; j, v) } & =((\phi i) a ; \alpha i, \mu)(b ; j, v)=\left((\phi i) a p_{\mu j} b ; \alpha i, v\right)=\lambda\left(a p_{\mu j} b ; i, v\right) \\
& =\lambda[(a ; i, \mu)(b ; j, v)],
\end{aligned}
$$

while otherwise both $[\lambda(a ; i, \mu)](b ; j, v)$ and $\lambda[(a ; i, \mu)(b ; j, v)]$ are zero. Since $\lambda(x y)=(\lambda x) y$ holds trivially when $x=0$ or $y=0$, we conclude that $\lambda \in \Lambda(S)$. It is clear that, for $\lambda$ defined by $(6), \mathbf{a} \lambda=(\alpha, \phi)$. Consequently $\mathbf{a}$ is an isomorphism of $\Lambda(S)$ onto $L(I, G)$. 
Now let $M$ be a non-empty set (elements of $M$ are denoted by $\mu, v, \ldots ; M$ stands for capital $\mu$ ) and let $G$ be a group. Set

with multiplication

$$
R(\mathrm{M}, G)=\left\{(\beta, \psi) \mid \beta \in W^{\prime}(\mathrm{M}), \beta \neq 0, \psi: \mathrm{d} \beta \rightarrow G\right\} \cup 0
$$

where

$$
\left.\begin{array}{l}
(\beta, \psi)\left(\beta^{\prime}, \psi^{\prime}\right)=\left(\beta \beta^{\prime}, \psi^{\prime \prime}\right) \text { if } \beta \beta^{\prime} \neq 0, \text { and } 0 \text { otherwise, } \\
(\beta, \psi) 0=0(\beta, \psi)=0
\end{array}\right\}
$$

$$
\mu \psi^{\prime \prime}=(\mu \psi)\left(\mu \beta \psi^{\prime}\right)
$$

Note that in $(\beta, \psi)$ both $\beta$ and $\psi$ are written as operators on the right. For every $\rho \in \mathrm{P}(S)$, $\rho \neq 0$, let

where

$$
\rho \mathbf{b}=(\beta, \psi), \quad \mathbf{0 b}=0,
$$

$$
(1 ; i, \mu) \rho=(\mu \psi ; i, \mu \beta) \text { if }(1 ; i, \mu) \rho \neq 0 .
$$

A proof entirely analogous to the proof of Theorem 1 establishes

THEOREM 2. The function $\mathrm{b}$ is an isomorphism of $\mathrm{P}(S)$ onto $R(\mathrm{M}, G)$.

We only state the analogue of (6) (used to prove that b is onto), namely, for $(\beta, \psi) \in R(M, G)$, define $\rho: S \rightarrow S$ by

$$
\begin{aligned}
& (a ; i, \mu) \rho=(a(\mu \psi) ; i, \mu \beta) \text { if } \mu \in \mathrm{d} \beta, \text { and } 0 \text { otherwise, } \\
& 0 \rho=0 .
\end{aligned}
$$

Then $\rho \in \mathrm{P}(S)$ and $\rho \mathrm{b}=(\beta, \psi)$.

For $\mathbf{a} \lambda=(\alpha, \phi), \rho \mathbf{b}=(\beta, \psi)$, we also have (see the proof of Theorem 1)

$$
\begin{aligned}
& \mathrm{d} \alpha=\{i \in I \mid \lambda(a ; i, \mu) \neq 0\}, \\
& \mathbf{d} \beta=\{\mu \in \mathbf{M} \mid(a ; i, \mu) \rho \neq 0\} .
\end{aligned}
$$

THEOREM 3. For $\mathbf{a} \lambda=(\alpha, \phi), \rho \mathrm{b}=(\beta, \psi), \lambda$ and $\rho$ are linked if and only if the following two conditions are satisfied:

$$
\begin{gathered}
i \in \mathrm{d} \alpha, p_{\mu(\alpha i)} \neq 0 \Leftrightarrow \mu \in \mathrm{d} \beta, p_{(\mu \beta) i} \neq 0 \quad(i \in I, \mu \in \mathrm{M}), \\
p_{\mu(\alpha i)}(\phi i)=(\mu \psi) p_{(\mu \beta) i} \text { if } \quad i \in \mathrm{d} \alpha, p_{\mu(\alpha i)} \neq 0 \quad(i \in I, \mu \in \mathrm{M})
\end{gathered}
$$

(cf. [1], (4.5)). Furthermore, if $\lambda$ and $\rho$ are linked, $\lambda=0$ if and only if $\rho=0$.

Proof. The first statement follows directly from the following calculation.

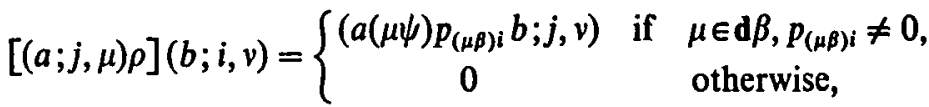

$$
\begin{aligned}
& (a ; j, \mu)[\lambda(b ; i, v)]=\left\{\begin{array}{cc}
\left(a p_{\mu(\alpha i)}(\phi i) b ; j, v\right) & \text { if } \quad i \in \mathrm{d} \alpha, p_{\mu(\alpha))} \neq 0 \\
0 & \text { otherwise. }
\end{array}\right.
\end{aligned}
$$


The second statement is easily checked.

Recall that, for any $\alpha \in W(I)$, rank $\alpha$ is the cardinality of the range $r \alpha$ of $\alpha$; similarly for $\beta \in W^{\prime}(\mathrm{M})$. To simplify notation, we define

$$
\operatorname{rank}(\alpha, \phi)=\operatorname{rank} \alpha, \quad \operatorname{rank}(\beta, \psi)=\operatorname{rank} \beta, \quad \operatorname{rank} 0=0 .
$$

TheOREM 4. For $0 \neq(\lambda, \rho) \in \Omega(S),(\lambda, \rho) \in \Pi(S)$ if and only if rank $\mathbf{a} \lambda=\operatorname{rank} \rho \mathbf{b}=1$.

Proof. Let $\mathbf{a} \lambda=(\alpha, \phi), \rho \mathbf{b}=(\beta, \psi)$.

Necessity. Let $0 \neq(\lambda, \rho) \in \Pi(S)$; by the definition of $\Pi(S)$,

$$
\lambda=\lambda_{(b ; j, v)}, \quad \rho=\rho_{(b ; j, v)}
$$

for some $(b ; j, v) \in S$. From (12) and (13), we obtain

For $i \in \mathrm{d} \alpha$, we have

$$
\begin{aligned}
& \mathrm{d} \alpha=\left\{i \in I \mid p_{v i} \neq 0\right\}, \\
& \mathrm{d} \beta=\left\{\mu \in \mathrm{M} \mid p_{\mu j} \neq 0\right\} .
\end{aligned}
$$

whence

$$
\lambda(a ; i, \mu)=(b ; j, v)(a ; i, \mu)=\left(b p_{v i} a ; j, \mu\right)=((\phi i) a ; \alpha i, \mu),
$$

$$
\phi i=b p_{v i}, \alpha i=j \quad(i \in \mathrm{d} \alpha) ;
$$

the last equality implies that rank $\alpha=1$. An analogous calculation proves:

$$
\mu \psi=p_{\mu j} b, \quad \mu \beta=v \quad(\mu \in \mathrm{d} \beta) ;
$$

again, the last equality implies that $\operatorname{rank} \beta=1$.

Sufficiency. By hypothesis, $\operatorname{rank} \alpha=\operatorname{rank} \beta=1$; so $\alpha i=j$ for all $i \in \mathrm{d} \alpha$ and some $j \in I$, similarly $\mu \beta=v$ for all $\mu \in \mathrm{d} \beta$ and some $v \in \mathrm{M}$.

Suppose that $p_{v i} \neq 0$; then for any $\mu \in \mathrm{d} \beta$ we have

$$
[(1 ; i, \mu) \rho](1 ; i, \mu)=(\mu \psi ; i, v)(1 ; i, \mu)=\left((\mu \psi) p_{v i} ; i, \mu\right) \neq 0,
$$

whence $\lambda(1 ; i, \mu) \neq 0$, which, by (12), implies that $i \in \mathrm{d} \alpha$.

Suppose next that $p_{v i}=0$. Let $\gamma \in \mathrm{M}$ be such that $p_{v j} \neq 0$. Then, since $p_{v i}=0$,

$$
[(1 ; i, \gamma) \rho](1 ; i, \mu)=(\gamma \psi ; i, v)(1 ; i, \mu)=0
$$

if $\gamma \in \mathbf{d} \hat{\beta}$, and is zero otherwise; it is therefore zero in all cases. Consequently

$$
0=(1 ; i, \gamma)[\lambda(1 ; i, \mu)]=(1 ; i, \gamma)(\phi i ; j, \mu)
$$

if $i \in \mathrm{d} \alpha$; this implies that $i \notin \mathrm{d} \alpha$, since $p_{y j} \neq 0$. This proves (18); (19) is established analogously.

Let $i \in \mathrm{d} \alpha, \mu \in \mathrm{d} \beta$; then, by (18) and (19), we have $p_{v i} \neq 0$ and $p_{\mu j} \neq 0$, and, by (15), $p_{\mu j}(\phi i)=(\mu \psi) p_{v i}$, whence $(\phi i) p_{v i}^{-1}=p_{\mu j}^{-1}(\mu \psi)$. Since $j$ and $v$ are fixed, in the last equation 
the left-hand side depends only on $i$ while the right-hand side depends only on $\mu$. Since $i \in \mathrm{d} \alpha$ and $\mu \in \mathrm{d} \beta$ are arbitrary, both sides must be equal to a constant, say $b$. Hence (20) and (21) are satisfied; since also (18) and (19) have been established, we may reverse the steps of the first part of the proof to see that (17) also holds. Thus $(\lambda, \rho) \in \Pi(S)$.

Corollary.

$$
\begin{aligned}
\Pi(S) & =\{(\lambda, \rho) \in \Omega(S) \mid \operatorname{rank} \mathbf{a} \hat{\lambda} \leqq 1, \operatorname{rank} \rho \mathbf{b} \leqq 1\} \\
& =\{(\lambda, \rho) \in \Omega(S) \mid \operatorname{rank} \mathbf{a} \lambda=\operatorname{rank} \rho \mathbf{b}=1\} \cup 0 .
\end{aligned}
$$

2. Matrix representation of translations. In [3, p. 116, exercise 3], the semigroup $\Lambda(S)$ is faithfully represented by the semigroup of all column-monomial $\mathrm{M} \times \mathbf{M}$-matrices over $G^{0}$; the case of a completely simple semigroup is treated in [2] (see also [1, \$4]). Analogously, $\mathrm{P}(S)$ is faithfully represented by the semigroup of all row-monomial $I \times I$-matrices over $G^{0}$. We now compare the construction in $\S 1$ with the representation by matrices.

Let $\mathscr{A}$ be the semigroup of all column-monomial (in every column at most one nonzero element) $I \times I$-matrices over $G^{0}$. For every $(\alpha, \phi) \in L(I, G)$, let

$$
\mathbf{c}(\alpha, \phi)=\left(a_{i j}\right), \quad \mathbf{c} 0=0
$$

(the last 0 is the matrix with all entries zero), where $\left(a_{i j}\right)$ is an $I \times I$-matrix defined by

$$
a_{i j}=\phi j \quad \text { if } j \in \mathrm{d} \alpha, \quad \alpha j=i, \quad \text { and } 0 \text { otherwise. }
$$

THEOREM 5. The function $\mathrm{c}$ is an isomorphism of $L(I, G)$ onto $\mathscr{A}$.

Proof. Since $\alpha$ is single-valued, $\left(a_{i j}\right) \in \mathscr{A}$ and thus c: $L(I, G) \rightarrow \mathscr{A}$. Let $\mathrm{c}(\alpha, \phi)=\left(a_{i j}\right)$, $\mathbf{c}\left(\alpha^{\prime}, \phi^{\prime}\right)=\left(a_{i j}^{\prime}\right)$. Then

$$
c\left((\alpha, \phi)\left(\alpha^{\prime}, \phi^{\prime}\right)\right)=\left\{\begin{array}{rlrl}
\mathbf{c}\left(\alpha \alpha^{\prime}, \phi^{\prime \prime}\right) & =\left(c_{i j}\right) & \text { if } \alpha \alpha^{\prime} \neq 0, \\
\mathbf{c} 0 & =0 & & \text { otherwise, }
\end{array}\right.
$$

where $\phi^{\prime \prime}$ is given by (3), and $c_{i j}=\phi^{\prime \prime} j$ if $j \in \mathrm{d}\left(\alpha \alpha^{\prime}\right), \alpha \alpha^{\prime} j=i$, and is 0 otherwise. Further,

where

$$
\mathbf{c}(\alpha, \phi) \mathbf{c}\left(\alpha^{\prime}, \phi^{\prime}\right)=\left(a_{i j}\right)\left(a_{i j}^{\prime}\right)=\left(d_{i j}\right) \text {, }
$$

$$
\begin{aligned}
d_{i j}=\sum_{k \in I} a_{i k} a_{k j}^{\prime} & =\left\{\begin{array}{cl}
(\phi k)\left(\phi^{\prime} j\right) \text { if } & k \in \mathrm{d} \alpha, \alpha k=i, j \in \mathrm{d} \alpha^{\prime}, \alpha^{\prime} j=k, \\
0 & \text { otherwise, }
\end{array}\right. \\
& =\left\{\begin{array}{cll}
\left(\phi \alpha^{\prime} j\right)\left(\phi^{\prime} j\right) & \text { if } & j \in \mathrm{d}\left(\alpha \alpha^{\prime}\right), \alpha \alpha^{\prime} j=i, \\
0 & \text { otherwise, }
\end{array}\right. \\
& =c_{i j} .
\end{aligned}
$$


Consequently $\mathbf{c}\left((\alpha, \phi)\left(\alpha^{\prime}, \phi^{\prime}\right)\right)=\mathbf{c}(\alpha, \phi) \mathbf{c}\left(\alpha^{\prime}, \phi^{\prime}\right)$, which obviously implies that $\mathbf{c}$ is a homomorphism. If follows easily that $\mathrm{c}$ is one-to-one.

If $\left(a_{i j}\right) \in \mathscr{A}$, for every $i, j \in I$, let $\alpha j=i$ and $\phi j=a_{i j}$ if $a_{i j} \neq 0$. It is easy to see that $(\alpha, \phi) \in L(I, G)$ and $\mathrm{c}(\alpha, \phi)=\left(a_{i j}\right)$. Hence $\mathrm{c}$ is an isomorphism of $L(I, G)$ onto $\mathscr{A}$.

Let $\mathscr{B}$ be the semigroup of all row-monomial $\mathrm{M} \times \mathrm{M}$-matrices over $G^{0}$, and for every $(\beta, \psi) \in R(\mathrm{M}, G)$, let

where

$$
(\beta, \psi) \mathbf{d}=\left(b_{\mu v}\right), \quad 0 \mathbf{d}=0
$$

$$
b_{\mu \nu}=\mu \psi \quad \text { if } \quad \mu \in \mathrm{d} \beta, \mu \beta=\nu, \quad \text { and is } 0 \text { otherwise. }
$$

A proof analogous to the proof of Theorem 5 establishes

THEOREM 6. The function $\mathrm{d}$ is an isomorphism of $R(\mathrm{M}, G)$ onto $\mathscr{B}$.

The next theorem should be compared with condition (D3) in [2].

THEOREM 7. Let $\lambda \in \Lambda(S)$ and $\rho \in \mathrm{P}(S)$, and let $A=\mathrm{ca} \lambda, B=\rho \mathrm{bd}$. Then $\lambda$ and $\rho$ are linked if and only if $P A=B P$.

Proof. Let $A=\left(a_{i j}\right), B=\left(b_{\mu v}\right)$; we have $P A=\left(c_{\mu j}\right), B P=\left(d_{\mu j}\right)$, where

$$
\begin{gathered}
c_{\mu j}=\sum_{i \in I} p_{\mu i} a_{i j}=\left\{\begin{array}{ccc}
p_{\mu(\alpha j)}(\phi j) & \text { if } & j \in \mathrm{d} \alpha, p_{\mu(\alpha j)} \neq 0, \\
0 & \text { otherwise, }
\end{array}\right. \\
d_{\mu j}=\sum_{v \in M} b_{\mu \nu} p_{v j}=\left\{\begin{array}{cll}
(\mu \psi) p_{(\mu \beta) j} & \text { if } & \mu \in \mathrm{d} \beta, p_{(\mu \beta) j} \neq 0, \\
0 & \text { otherwise. }
\end{array}\right.
\end{gathered}
$$

The theorem now follows by Theorem 3.

It follows from the above discussion that rank $(\alpha, \phi)(=\operatorname{rank} \alpha)$ is the number of nonzero rows of $\mathrm{c}(\alpha, \phi)$; similarly rank $(\beta, \psi)(=\operatorname{rank} \beta)$ is the number of non-zero columns of $(\beta, \psi) \mathrm{d}$.

3. Uniqueness of linked translations. In this section we discuss the conditions under which to every left translation of $S$ there is at most one linked right translation, and related questions. By $\imath_{A}$ denote the identity mapping of a non-empty set $A$, and by $\phi_{A}$ (also $\psi_{A}$ ) denote the mapping on $A$ onto the identity 1 of the group $G$; note that $\left(l_{I}, \phi_{I}\right)$ and $\left(l_{M}, \psi_{M}\right)$ are identities of $L(I, G)$ and $R(\mathrm{M}, G)$, respectively. To simplify notation, in this section we identify $\Lambda(S)$ and $\mathrm{P}(S)$ with $L(I, G)$ and $R(\mathrm{M}, G)$, respectively.

THEOREM 8. On $S$ the following conditions are equivalent: 
(a) To every left translation there is at most one linked right translation;

(b) the only right translation linked to the identity transformation as a left translation is the identity transformation;

(c) $p_{\mu i}=c p_{v i}$ for some $c \in G$ and all $i \in I$ implies that $\mu=v$;

(d) to every inner left translation there is at most [exactly] one linked inner right translation;

(e) $S$ is right reductive.

Proof. $(a) \Rightarrow(b)$. Obvious.

on $\mathbf{M}$ by

$(b) \Rightarrow(c)$. Suppose that $p_{\mu i}=c p_{v i}$ for some $c \in G$ and all $i \in I$. Define $\beta$ and $\psi$

Then

$$
\gamma \beta=\left\{\begin{array}{ll}
\nu & \text { if } \gamma=\mu, \\
\gamma & \text { otherwise }
\end{array}\right\} ; \quad \gamma \psi=\left\{\begin{array}{ll}
c & \text { if } \gamma=\mu, \\
1 & \text { otherwise }
\end{array}\right\}
$$

$$
p_{\gamma i}=\left\{\begin{array}{ll}
c p_{v i} & \text { if } \gamma=\mu, \\
p_{y i} & \text { otherwise }
\end{array}\right\} ; \quad p_{(\gamma \beta) i}=\left\{\begin{array}{ll}
p_{v i} & \text { if } \gamma=\mu, \\
p_{\gamma i} & \text { otherwise }
\end{array}\right\} ;
$$

thus $p_{y i} \neq 0 \Leftrightarrow p_{(\gamma \beta) i} \neq 0$, and, if $p_{y i} \neq 0$,

$$
(\gamma \psi) p_{(\gamma \beta) i}=\left\{\begin{array}{ll}
c p_{v i} & \text { if } \gamma=\mu, \\
p_{\gamma i} & \text { otherwise }
\end{array}\right\}=p_{y i} 1 .
$$

By Theorem 3, it follows that $\left(l_{I}, \phi_{I}\right)$ and $(\beta, \psi)$ are linked, which by hypothesis implies $\beta=l_{\mathrm{M}}, \psi=\psi_{\mathrm{M}}$. But then $\mu=\mu l_{\mathrm{M}}=\mu \beta=v$.

$(c) \Rightarrow(e)$. Suppose that

$$
(a ; i, \mu)(x ; k, \gamma)=(b ; j, v)(x ; k, \gamma) \quad((x ; k, \gamma) \in S) .
$$

For $p_{\mu k} \neq 0$ and $x=1$, (22) yields $a p_{\mu k}=b p_{v k}$; whence $p_{\mu k}=a^{-1} b p_{v k}$. It also follows from (22) that $p_{\mu k} \neq 0 \Leftrightarrow p_{v k} \neq 0$, so that $p_{\mu k}=a^{-1} b p_{v k}$ is valid for all $k \in I$. By (c), we have $\mu=v$, which together with $p_{\mu k}=a^{-1} b p_{v k}$ implies $a=b$. Since (22) also implies $i=j$, we have proved that $(a ; i, \mu)=(b ; j, v)$.

$(e) \Rightarrow(a)$. If $\lambda$ is linked to $\rho$ and $\rho^{\prime}$, then, for all $x, y \in S, x(\lambda y)=(x \rho) y=\left(x \rho^{\prime}\right) y$, whence $x \rho=x \rho^{\prime}$, since $S$ is right reductive. Thus $\rho=\rho^{\prime}$.

$(a) \Rightarrow(d)$. This follows from the fact that $\left(\lambda_{y}, \rho_{y}\right) \in \Omega(S)$ for any $y \in S$.

$(d) \Rightarrow(a)$. Suppose that $\lambda$ is linked to $\rho$ and $\rho^{\prime}$. Then, for all $x, y \in S, x(\lambda y)=(x \rho) y$ $=\left(x \rho^{\prime}\right) y$, so that $\lambda_{x \rho}=\lambda_{x \rho^{\prime}}$. But then $\lambda_{x \rho}$ is linked to both $\rho_{x \rho}$ and $\rho_{x \rho^{\prime}}$, which, by $(d)$ implies that $\rho_{x \rho}=\rho_{x \rho^{\prime}}$. Consequently, for all $x, y \in S, y(x \rho)=y\left(x \rho^{\prime}\right)$, that is, $(y x) \rho=(y x) \rho^{\prime}$. Since $S^{2}=S$, we have $\rho=\rho^{\prime}$. 
Remark. It follows from the proof of Theorem 8 that $(a) \Rightarrow(d)$ and $(e) \Rightarrow(a)$ in any semigroup while $(d) \Rightarrow(a)$ in any semigroup $S$ for which $S^{2}=S$.

Some of the conditions appearing in Theorem 8 may be phrased as follows:

(a) the projection mapping $\Omega(S) \rightarrow \Lambda(S)$ is one-to-one;

(c) no two different rows of $P$ are left proportional (cf. [3, p. 119, exercise 2]);

(d) the projection mapping $\Pi(S) \rightarrow \Gamma(S)$ (inner left translations) is one-to-one;

(e) the mapping $x \rightarrow \lambda_{x}$ is one-to-one.

Since the mappings in $(a),(d),(e)$ are always homomorphisms, we have

CoROLlaRY. If $S$ is right reductive, then

$$
S \cong \Gamma(S) \cong \Pi(S), \quad \tilde{\Lambda}(S) \cong \Omega(S),
$$

where $\tilde{\Lambda}(S)$ is the projection of $\Omega(S)$ into $\Lambda(S)$.

THEOREM 9. On $S$ the following conditions are equivalent:

(a) If $(\alpha, \phi)$ is linked to $(\beta, \psi)$ and to $\left(\beta^{\prime}, \psi\right)$, then $\beta=\beta^{\prime}$;

(b) $\left(\left(l_{I}, \phi_{I}\right),\left(\beta, \psi_{\mathrm{M}}\right)\right) \in \Omega(S)$ implies $\beta=\imath_{\mathrm{M}}$;

(c) $p_{\mu i}=p_{v i}$ for all $i \in I$, implies $\mu=v$.

Proof. $(a) \Rightarrow(b)$. This follows from $\left(\left(l_{I}, \phi_{I}\right),\left(l_{M}, \psi_{M}\right)\right) \in \Omega(S)$.

$(b) \Rightarrow(c)$. Suppose that $p_{\mu i}=p_{v i}$ for all $i \in I$ and define $\beta$ as in the proof of Theorem 8 $((b) \Rightarrow(c))$. Then $p_{\gamma i}=p_{(\gamma \beta) i}$ for all $i \in I$, which by Theorem 3 implies that $\left(\left(l_{I}, \phi_{I}\right),\left(\beta, \psi_{M}\right)\right)$ $\in \Omega(S)$. But then the hypothesis implies that $\beta=\beta_{\mathrm{M}}$, whence $\mu=v$.

$(c) \Rightarrow(a)$. Suppose that $(\alpha, \phi)$ is linked to $(\beta, \psi)$ and to $\left(\beta^{\prime}, \psi\right)$. From the very definition of $(\beta, \psi)$ and $\left(\beta^{\prime}, \psi\right)$, it follows that $\mathbf{d} \beta=\mathbf{d} \beta^{\prime}$. Applying Theorem 3 twice, we have, for any $\mu \in \mathrm{d} \beta$,

$$
\begin{gathered}
p_{(\mu \beta) i} \neq 0 \Leftrightarrow p_{\left(\mu \beta^{\prime}\right) i} \neq 0 \quad(i \in I), \\
(\mu \psi) p_{(\mu \beta) i}=(\mu \psi) p_{\left(\mu \beta^{\prime}\right) i} \text { if } p_{(\mu \beta) i} \neq 0 \quad(i \in I),
\end{gathered}
$$

whence $p_{(\mu \beta) i}=p_{\left(\mu \beta^{\prime}\right) t}$ for all $i \in I$. The hypothesis then implies that $\mu \beta=\mu \beta^{\prime}$, and, since $\mu \in \mathbf{d} \beta$ is arbitrary, $\beta=\beta^{\prime}$.

Note that, if $(\alpha, \phi)$ is linked to $(\beta, \psi)$ and $\left(\beta, \psi^{\prime}\right)$, then Theorem 3 implies that $\psi=\psi^{\prime}$. "Left-right" duality yields the corresponding theorems for right translations.

4. Some properties of $L(I, G)$ and $R(\mathrm{M}, G)$. We finally turn to some simple properties of $L(I, G)$ and $R(\mathrm{M}, G)$ (that is, of $\Lambda(S)$ and $\mathrm{P}(S)$, respectively). 
Proposition 1. The identity mapping of the set $L(I, G)$ onto the set $R(I, G)$ is an antiisomorphism if and only if $G$ is commutative.

Proof. Denote by * and o the multiplications in $L(I, G)$ and $R(I, G)$, respectively.

Necessity. By hypothesis we have

$$
(\alpha, \phi) *\left(\alpha^{\prime}, \phi^{\prime}\right)=\left(\alpha^{\prime}, \phi^{\prime}\right) \circ(\alpha, \phi) \quad\left((\alpha, \phi),\left(\alpha^{\prime}, \phi^{\prime}\right) \in L(I, G)\right) .
$$

Let $a, b \in G$; fix $i \in I$ and let $\alpha=\alpha^{\prime}: i \rightarrow i, \phi: i \rightarrow a, \phi^{\prime}: i \rightarrow b$. Then

$$
\begin{array}{ll}
(\alpha, \phi) *\left(\alpha^{\prime}, \phi^{\prime}\right)=\left(\alpha, \phi^{\prime \prime}\right), & \phi^{\prime \prime} i=\left(\phi \alpha^{\prime} i\right)\left(\phi^{\prime} i\right)=a b, \\
\left(\alpha^{\prime}, \phi^{\prime}\right) \circ(\alpha, \phi)=\left(\alpha, \psi^{\prime \prime}\right), & i \psi^{\prime \prime}=\left(i \phi^{\prime}\right)\left(i \alpha^{\prime} \phi\right)=b a,
\end{array}
$$

which by (23) implies that $a b=b a$.

Sufficiency. We have

$$
\begin{aligned}
& (\alpha, \phi) *\left(\alpha^{\prime}, \phi^{\prime}\right)=\left(\alpha \alpha^{\prime}, \phi^{\prime \prime}\right) \text { if } \alpha \alpha^{\prime} \neq 0, \text { and } 0 \text { otherwise, } \\
& \left(\alpha^{\prime}, \phi^{\prime}\right) \circ(\alpha, \phi)=\left(\alpha^{\prime} \alpha, \psi^{\prime \prime}\right) \text { if } \alpha^{\prime} \alpha \neq 0, \text { and } 0 \text { otherwise. }
\end{aligned}
$$

Further

$$
\left(\alpha \alpha^{\prime}\right) i=\alpha\left(\alpha^{\prime} i\right)=\alpha\left(i \alpha^{\prime}\right)=\left(i \alpha^{\prime}\right) \alpha=i\left(\alpha^{\prime} \alpha\right),
$$

and, by the commutativity of $G$,

$$
\phi^{\prime \prime} i=\left(\phi \alpha^{\prime} i\right)\left(\phi^{\prime} i\right)=\left(\phi^{\prime} i\right)\left(\phi \alpha^{\prime} i\right)=\left(i \phi^{\prime}\right)\left(i \alpha^{\prime} \phi\right)=i \psi^{\prime \prime} .
$$

Comparing (24), (25), (26), and (27), we obtain (23).

Let $A$ be any set and $\sigma$ an equivalence relation on $A$. We say that a set $C$ is a crosssection of $\sigma$ if $C$ is a subset of $A$ intersecting every class of $\sigma$ exactly once.

Proposition 2. The semigroups $L(I, G)$ and $R(\mathrm{M}, G)$ are regular.

Proof. We consider only $L(I, G) ; R(\mathrm{M}, G)$ is treated analogously. Let $(\alpha, \phi) \in L(I, G)$ and let $C$ be any cross-section of the equivalence relation on $\mathrm{d} \alpha$ induced by $\alpha$. Define $\alpha^{\prime}$ and $\phi^{\prime}$ as follows: Let $\mathrm{d} \alpha^{\prime}=\mathrm{r} \alpha, \alpha^{\prime}(\alpha i)=j$ if $\alpha i=\alpha j$ and $j \in C, \phi^{\prime}(\alpha i)=\left(\phi \alpha^{\prime} \alpha i\right)^{-1}$. Then $\alpha \alpha^{\prime} \alpha=\alpha$ and

$$
\left(\phi \alpha^{\prime} \alpha i\right)\left(\phi^{\prime} \alpha i\right)(\phi i)=\left(\phi \alpha^{\prime} \alpha i\right)\left(\phi \alpha^{\prime} \alpha i\right)^{-1}(\phi i)=\phi i,
$$

so that $(\alpha, \phi)=(\alpha, \phi)\left(\alpha^{\prime}, \phi^{\prime}\right)(\alpha, \phi)$.

Proposition 3. Let $A$ be a non-empty subset of $I, \sigma$ an equivalence relation on $A, C$ any cross-section of $\sigma$. Define $\alpha: A \rightarrow C$ and $\phi: A \rightarrow G$ as follows: $\alpha i=j$ if $i \sigma j$ and $j \in C, \phi i=1$ if $i \in C$, otherwise arbitrary. Then $(\alpha, \phi)$ is a non-zero idempotent of $L(I, G)$ and all non-zero idempotents of $L(I, G)$ can be constructed in this fashion. 
Proof. It is easily verified that the above construction yields a non-zero idempotent of $L(I, G)$. Conversely, if $(\alpha, \phi)$ is a non-zero idempotent of $L(I, G)$, then let $A=\mathrm{d} \alpha, C=\mathrm{r} \alpha$, $\sigma$ be the equivalence on $A$ induced by $\alpha$. From $\alpha i=\alpha(\alpha i)$, it follows that $\alpha i=j$ if $i \sigma j$ and $j \in C$; from $\phi i=(\phi \alpha i)(\phi i)$, it follows that $\phi i=1$ if $i \in C$.

\section{REFERENCES}

1. A. H. Clifford, Semigroups admitting relative inverses, Annals of Math. 42 (1941), 1037-1049.

2. A. H. Clifford, Extensions of semigroups, Trans. Amer. Math. Soc. 68 (1950), 165-173.

3. A. H. Clifford and G. B. Preston, The algebraic theory of semigroups, Vol. I, Math. Surveys No. 7, Amer. Math. Soc. (Providence, R. I., 1961).

4. L. M. Gluskin, Ideals of transformation semigroups, Mat. Sb. 47(1959), 111-130 (Russian).

5. L. M. Gluskin, Semigroups and rings of endomorphisms of linear spaces, Izv. Akad. Nauk SSSR, ser. mat. 23 (1959), 841-870 (Russian).

6. L. M. Gluskin, Ideals of semigroups, Mat. $S b$. 55, (1961) 421-448 (Russian).

7. Reikichi Yoshida, Ideal extensions of semigroups and compound semigroups, Mem. Res. Inst. Sci. Eng., Ritumeikan Univ. 13 (1965), 1-8.

The Pennsylvania State University

University Park, Pennsylvania 